\title{
Calculation of nonlinear envelopes in beam expanders
}

\author{
F. Méot* \\ CEA, DSM/DAPNIA/SEA, 91191 Saclay, France
}

T. Aniel ${ }^{\dagger}$

CEA, DSM/DRFC/SPPF, 13108 Cadarache, France

(Received 16 August 1999; published 12 October 2000)

\begin{abstract}
An analytical method for calculating nonlinear envelopes in beam expanders is presented. It is illustrated by implementation in a matrix transport code and accompanied with various considerations on expander line design and transverse beam tail confinement effects.
\end{abstract}

PACS numbers: 29.27.Fh

\section{INTRODUCTION}

Beam expanders are transport lines that provide transverse expansion of particle beam by means of first order optical elements associated with transverse density uniformization by nonlinear lenses. They are supposed to be placed at the high energy end of LINAC installations devoted to such applications as nuclear waist transmutation, spallation neutron sources, medical irradiation, etc. [1-3].

An analytical method for the calculation of nonlinear envelopes in beam expanders is presented, based on earlier works regarding transverse uniformization and related optical tuning of the beam line [4-7]. The material can be easily implemented in regular matrix transport codes, thereby allowing efficient use for beam line design and optimization with such features as mastering of particle loss and transverse aperture of optical elements.

This paper is organized in the following way: In Sec. II, the basis analytical material is introduced and applied for illustration to an existing design through implementation in the matrix code BETA [8]. Section III addresses coupling issues as related to momentum spread and geometrical aberrations likely to alter previous considerations. Section IV shows how nonlinear lenses, apart from uniformization, can also be used for obtaining transverse beam tail confinement.

\section{NONLINEAR ENVELOPES}

The calculation of nonlinear envelopes in a beam expander (Sec. II B) is based on analytical material first derived in Ref. [5] that is briefly recalled in Sec. II A; the Appendices provide additional details.

\section{A. Preamble}

Consider a beam expander line with first order optics assured by an assembly of quadrupoles ${ }^{1}(\mathrm{Q} 1-\mathrm{Q} 7)$ as shown

\footnotetext{
*Email address: fmeot@cea.fr

†Email address: taniel@cea.fr
}

in Fig. 1; two nonlinear lenses, $\mathrm{OH}$ and $\mathrm{OV}$, are devoted to the independent uniformization (see Appendix A for details) of horizontal and vertical transverse beam densities at a target (not shown in the figure) situated at the right end of the line $13.6 \mathrm{~m}$ downstream of a final small bend (BEND) that accessorily allows radiation shielding. At arbitrary location $s$ downstream of a uniformization lens, the transverse density can be obtained from [5] (see Appendix B)

$$
g(y(s))=\sum_{i=1}^{N} \frac{f\left[y_{l i}^{\prime}(y(s))\right]}{\left|\sum_{p=0}^{n}(2 p+1) \lambda_{2 p+1}(s) y_{l i}^{\prime 2 p}(y(s))\right|},
$$

where coordinates $y$ and $y^{\prime}=d y / d s$ stand for either $x, x^{\prime}$ (horizontal motion) or $z, z^{\prime}$ (vertical motion), the index

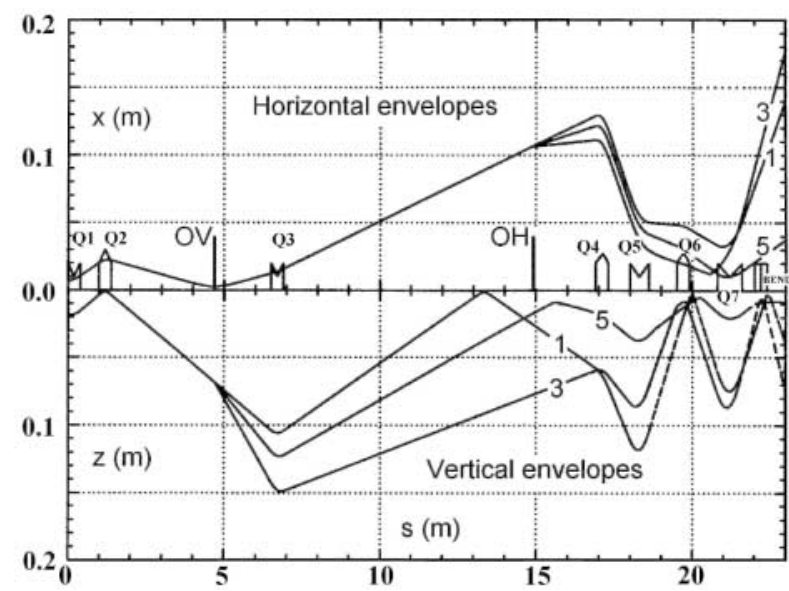

FIG. 1. Typical expander geometry, together with the horizontal envelopes $X_{1,3,5}(s)$ (upper curves) and the vertical envelopes $Z_{1,3,5}(s)$ (lower curves).

\footnotetext{
${ }^{1}$ This design is inspired from Ref. [3], very close to that considered for uniformization of a $700 \mathrm{MeV}$ proton beam in Ref. [5], where one may refer for more details on the geometry and magnet strengths.
} 
$l$ indicates quantities taken at the uniformization lens of concern, and $y_{l i}^{\prime}(i=1, N)$ are the $N \leq 2 n+1$ real roots of the polynomial equation

$$
y(s)=\sum_{p=0}^{n} \lambda_{2 p+1}(s) y_{l}^{\prime 2 p+1},
$$

wherein $\quad \lambda_{1}(s)=r_{l} \sqrt{\beta(s) / \beta_{l}} \cos (\Delta \phi), \quad \lambda_{2 p+1}(s)=$ $-K_{2 p+1} L r_{l}^{2 p+1} \sqrt{\beta(s) \beta_{l}} \sin (\Delta \phi), r_{l}=-\beta_{l} / \alpha_{l}, \quad \beta$ and $\alpha=-\beta^{\prime} / 2$ are the betatron functions, $\Delta \phi=\phi(s)-$ $\phi_{l}$ is the phase advance, and $K_{2 p+1} L(p=1, n)$ are the integrated strengths of the $n$ odd-order nonlinear components present in the lens ${ }^{2}$ (see Appendix C).

\section{B. Envelope equation}

\section{Integral equation}

First order beam optics normally deals with envelopes in terms of the transport of the optical functions $\alpha(s)$, $\beta(s), \gamma=\left(1+\alpha^{2}\right) / \beta, D(s)$ (dispersion function) while rms beam size in dispersion free regions is given by $\sigma(s)=\sqrt{\beta(s) \epsilon / \pi}$ with $\epsilon / \pi=$ beam emittance. Transverse apertures (chamber, bore, etc.) are usually defined in terms of the $k \sigma$ envelope, with $k$ being a few units depending on the loss tolerances. The parameter of concern in this respect is the loss rate $\tau$, quantified as the relative population beyond the $k \sigma$ width of a Gaussian distribution; that is, $\tau=1-\operatorname{erf}(k \sigma)$. In a beam expander the nonlinearities introduced by the uniformization lens are so strong that it is no longer relevant to address $\tau$ in terms of the $k \sigma$ envelope because the transverse density $g(y(s))$ [Eq. (1)] is much too far from Gaussian. In fact, given a tolerable loss rate $\tau$ (e.g., of the order of $10^{-7}-10^{-9}$ in high energy, high intensity installations), the $(2 n+1)$ th order nonlinear envelope $Y_{2 n+1}(s)$ is obtained by solving

$$
\int_{0}^{Y_{2 n+1}(s)} g(y) d y=1-\tau
$$

with $g(y)$ from Eq. (1) or, equivalently, by solving for $Y_{2 n+1}(s)$ the integral equation [drawn from Eq. (B1) in Appendix B]

$$
\sum_{i=1}^{N} \int_{y_{l i}^{\prime}(y=0)}^{y_{l i}^{\prime}\left[y=Y_{2 n+1}(s)\right]} f\left(y_{l i}^{\prime}\right)\left|d y_{l i}^{\prime}\right|=1-\tau .
$$

\section{Gaussian incoming beam}

In conventional optics methods the incoming beam density is usually considered Gaussian; namely, $f\left(y_{l}^{\prime}\right)=\exp \left(-y_{l}^{\prime 2} / 2 \sigma_{y_{l}^{\prime}}^{2}\right) / \sigma_{y_{l}^{\prime}} \sqrt{2 \pi}$ with $\sigma_{y_{l}^{\prime}}=$

\footnotetext{
${ }^{2}$ In regular notations, strengths write $K_{2 p+1}=B_{\text {pole }} / a_{\text {pole }}^{2 p+1} B \rho$ with $B_{\text {pole }}=$ field at pole tip, $a_{\text {pole }}=$ radius at pole tip, and $B \rho=$ beam rigidity.
}

$\sqrt{\gamma_{y, l} \epsilon_{y} / \pi}$, in which case the integral Eq. (4) above can easily be simplified into the more convenient form

$$
\operatorname{sgn}\left(\lambda_{1}\right) \sum_{i=1}^{N}(-1)^{i} \operatorname{erf}\left[y_{l i}^{\prime}\left(Y_{2 n+1}(s)\right)\right]=1-\tau .
$$

\section{Application}

This analytical material has been installed in a matrix transport code (BETA [8]), as illustrated in Fig. 1 which shows envelopes in the linear (curve 1), octupole (curve 3), and octupole + dodecapole (curve 5) cases for a loss $\tau=$ $10^{-7}$. The computation procedure is as follows: First, the roots $y_{l i}^{\prime}\left(y=Y_{2 n+1}(s)\right)$ of Eq. (2) are calculated (analytically in the case $2 n+1=3$ and numerically for $2 n+$ $1=5$ ). Next, given $\tau$, Eq. (5) is solved numerically for $Y_{2 n+1}(s)$ by a stepwise method. That procedure has the merit of additionally providing the local transverse density $g(y(s))$ at arbitrary $s$, as illustrated in Fig. 2.

In order to validate that material, Fig. 3 provides a comparison with envelopes generated by numerical tracking of 200 particles launched on a $5.3 \sigma$ invariant: The excellent behavior of the analytical model (solid lines) appears in that analytical envelopes coincide with the extreme excursions of the tracking (dotted trajectory arcs within optical elements).

It is worth noting the strong differences in the transverse beam size and, as well, the confinement generally induced by the octupole + dodecapole configuration compared with both the sole octupole and linear cases. In terms of the transverse aperture of the optical elements along the beam line, it means that one can afford much smaller bore diameters when using a combined octupole + dodecapole lens. In this respect, as discussed in Sec. IV, the confinement might still improve with an additional seventh order component (16-pole multipole) that would introduce

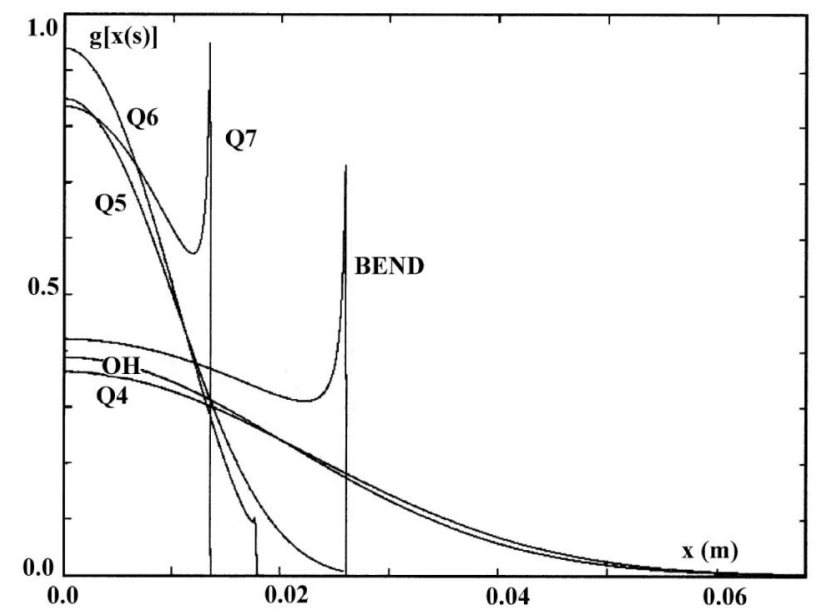

FIG. 2. Transverse horizontal beam density profiles $g(x)$ [Eq. (1) or, as well, a subproduct of the numerical solution of Eq. (5)] at various optical elements along the beam line shown in Fig. 1, given a sole octupole component $(n=1)$ at $\mathrm{OH}$. 

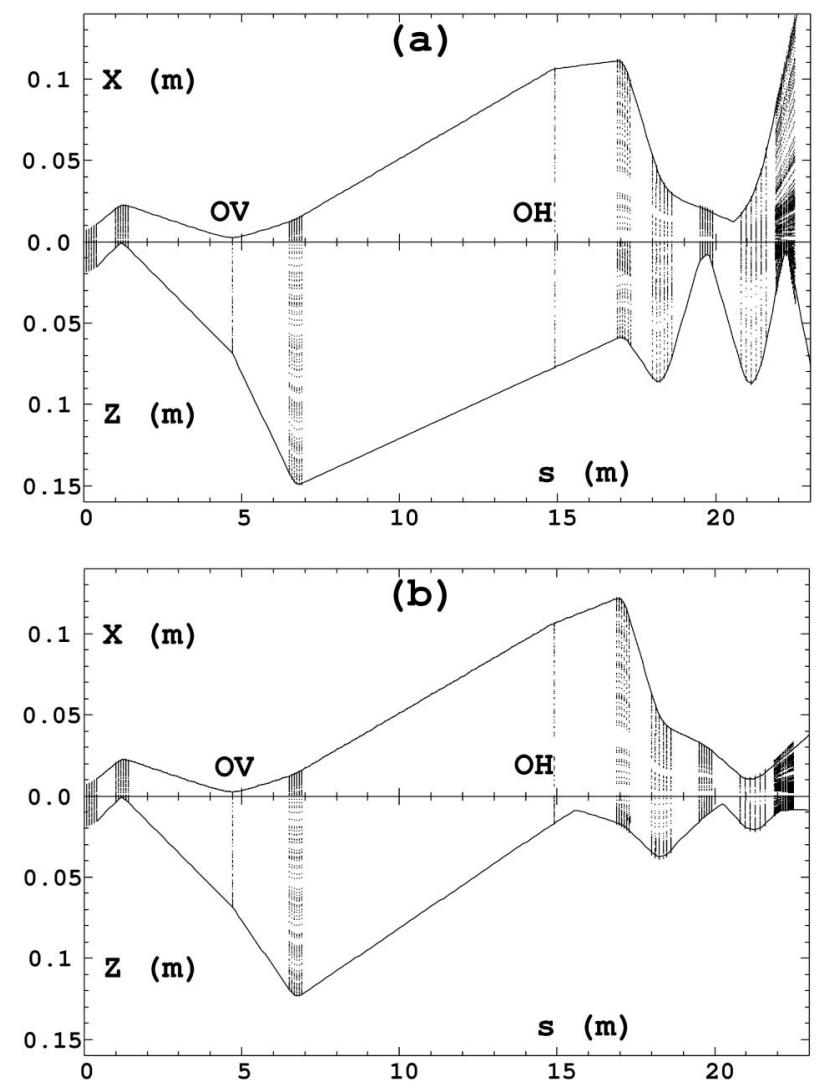

FIG. 3. Nonlinear third order $X_{3}, Z_{3}(s)$ [graph (a)] and fifth order $X_{5}, Z_{5}(s)$ [graph (b)] beam envelopes [solid lines; after Eq. (5)], and comparison with particle tracking (dotted trajectories).

a further seventh order loss limit; this could be of interest if very low loss rates (say, $\tau<10^{-9}$ ) were to be achieved.

\section{COUPLING}

Presence of coupling may sensibly affect the validity of the envelope Eq. (5). Second order is introduced by the quadrupoles and is purely chromatic (as long as such perturbation as a weak bend, e.g., the $6^{\circ}$ deviation "BEND" shielding element in Fig. 1, is neglected). It is shown first in Sec III A that it can be taken into account within the formalism described above, whereas Sec. III B brings arguments concerning higher order geometrical coupling.

\section{A. Momentum coupling}

The issue of momentum coupling can be addressed in terms of convolution of the variables $y(s)$ and $\delta$ (momentum deviation) as follows [4]. Equation (2) takes the modified form

$$
y^{*}(s)=\left(\frac{y}{y \delta}\right) y_{l} \delta+\left(\frac{y}{y^{\prime} \delta}\right) y_{l}^{\prime} \delta+\sum_{p=0}^{n} \lambda_{2 p+1}(s) y_{l}^{\prime 2 p+1},
$$

where $(y / y \delta)$ and $\left(y / y^{\prime} \delta\right)$ are the second order transport chromatic coefficients ${ }^{3}$ from the lens down to $s$. By introducing the rms value $\sigma_{y_{l}^{\prime}}$ of $y_{l}^{\prime}$ one gets

$$
y^{*}(s)=\xi \sigma_{y_{l}^{\prime}} \delta+\sum_{p=0}^{n} \lambda_{2 p+1}(s) y_{l}^{\prime 2 p+1} .
$$

This equation tells that, assuming that $y_{l}^{\prime}$ and $\delta$ are independent variables, the density $g^{*}\left(y^{*}(s)\right)$ is obtained by convolution of the separate densities, respectively, $g(y(s))$ [Eq. (1)] and $h(\delta)$, of the two independent variables $\sum_{p=0}^{n} \lambda_{2 p+1}(s) y_{l}^{\prime 2 p+1}$ and $\xi \sigma_{y_{l}^{\prime}} \delta$; in other words,

$$
g^{*}\left(y^{*}(s)\right)=g(y(s)) * h\left(\xi \sigma_{y_{l}^{\prime}} \delta\right) .
$$

Regular Fourier or Monte Carlo methods achieve this convolution. The ensuing perturbed envelope $Y_{2 n+1}^{*}(s)$ is thus obtained by numerical solution of

$$
\int_{0}^{Y_{2 n+1}^{*}(s)} g^{*}(y) d y=1-\tau .
$$

Note that, in order to avoid numerical calculation problems associated with possible lateral discontinuities of the first kind in $g(y)$ such as observed in Fig. 2, it is wise to work out integral Eq. (9) under the form of Eq. (4), which instead involves the presumably smooth (bell shaped) density $f\left(y_{l}^{\prime}\right)$.

A special case is in the calculation of $g^{*}(y)$ at target location which shows that significant momentum spread in the beam is beneficial to uniformization in that it damps the side peaks ${ }^{4}$ of $g(y)$ as shown in Fig. 4. Yet this is sensible only if the width $\sigma_{\delta}$ of $h(\delta)$ compares with that of those peaks, which is written $\left(y / y^{\prime} \delta\right) \sigma_{y_{l}^{\prime}} \sigma_{\delta} \approx$ a fraction of $y_{M}$. This can be understood in the following way: given that $y_{l}=r_{l} y_{l}^{\prime}$ [Eq. (A1) in Appendix A] and noting $\xi=(y / y \delta) r_{l}+\left(y / y^{\prime} \delta\right)$, Eq. (6) transforms to

$$
y^{*}(s)=\left(\lambda_{1}+\xi \delta\right) y_{l}^{\prime}+\sum_{p=1}^{n} \lambda_{2 p+1}(s) y_{l}^{\prime 2 p+1},
$$

so that momentum spread causes a blur of the uniformized beam width that is now written as

$$
y_{M}^{*}=\frac{2}{3}\left(\lambda_{1}+\xi \delta\right)\left(-\frac{\lambda_{1}+\xi \delta}{3 \lambda_{3}}\right)^{1 / 2} .
$$

For instance, taking $\left(x / x^{\prime} \delta\right) \sigma_{x_{l}^{\prime}} \sigma_{\delta}=10^{-2} x_{M}$ and with $\left(x / x^{\prime} \delta\right) \approx 10^{3}$ at target in the structure of Fig. 1 while $\sigma_{x_{l}^{\prime}} \approx 10^{-3} \mathrm{rad}$ is the horizontal beam divergence at the nonlinear lens $\mathrm{OH}$, it ensues that, given a Gaussian momentum distribution $h(\delta)=e^{-\delta^{2} / 2 \sigma_{\delta}^{2}} / \sigma_{\delta} \sqrt{2 \pi}$ one needs

\footnotetext{
${ }^{3}$ The transport coefficients $\left(x, x^{\prime} / z \delta\right),\left(x, x^{\prime} / z^{\prime} \delta\right)$ are neglected, which is reasonable in the present example; whether this is justified or not is certainly design dependent and needs attention.

${ }^{4}$ With an effect somewhat similar to that of an additional dodecapole component; see Fig. 11 in Ref. [5].
} 


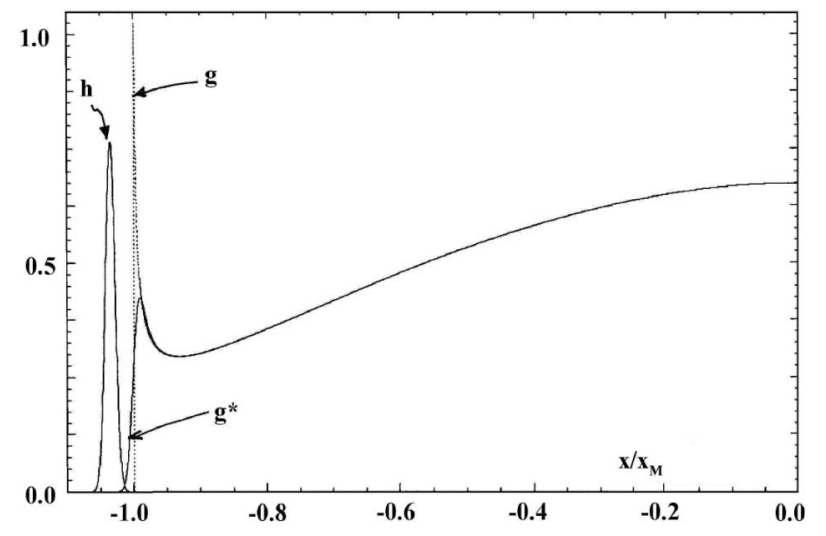

FIG. 4. Damping of the side peaks in $g^{*}(x)=g * h(x)$ due to Gaussian momentum spread $h(\delta)$.

momentum spread $\sigma_{\delta} \approx 10^{-2}$ in order to get sensible damping 5; this is illustrated in Fig. 4, which shows $g^{*}(x)$ at target (solid line), together with the unperturbed $g(x)$ for comparison [dotted line; Eq. (1) with $n=1$, octupole alone at $\mathrm{OH}]$ and the momentum distribution $h(\delta)$.

\section{B. Geometrical coupling}

Envelope equations in Sec. II B assume uncoupled $x$ and $z$ motions. This may not always be true, as in the example of Sec. IIC. It has been noticed [5,7] that this design does ensure the independence of the $z$ motion whatever the horizontal beam emittance $\epsilon_{x} / \pi$, whereas the reciprocal is not quite satisfied downstream of Q4; this was attributed to slight third order or higher order geometrical coupling introduced by the final set of quadrupoles Q4-Q7.

One way to address such issues is to include higher order in the analytical formalism and treat coupling in terms of the convolution of independent variables ${ }^{6}$, similar to what is done in Sec. III A. Another method is to refine the optical design in order to ensure best uncoupling, which is presumably preferable because during operation one certainly desires to have independent control of the $x$ and $z$ uniformization and transverse tails; a strong concern in this respect is the minimization of the waists in the uniformization lenses, which is a sine qua non condition for the functioning of the expander [5] and is a first order optics issue in the first lens (OV) and a nonlinear optics issue in the second lens $(\mathrm{OH})$ due to the latter being located downstream of the former.

Note that this effect of OV displacing the horizontal waist away from $\mathrm{OH}$ appears clearly in Figs. 1 and 3 (it can, in particular, be noticed that the best tuning at $\mathrm{OH}$ in

\footnotetext{
${ }^{5}$ Intense peaks would possibly induce harmful thermal effects. This suggests using dedicated rf cavity upstream of the beam expander in order to increase $\sigma_{\delta}$ in case the natural momentum spread of the beam would be too low.

${ }^{6}$ It can also be useful to refer to Ref. [9] for a discussion of convolution as related to coupling.
}

this respect is in the octupole + dodecapole case and corresponds to the goal of fifth-order uniformization at target in the present design. The issue of precise positioning of waists for getting sufficient uncoupling can be addressed in terms of the minimization of the nonlinear envelopes (for instance at $\mathrm{OH}$ in the present case) by means of the formalism developed above from which numerical fitting methods can be worked out for implementation in regular matrix codes.

\section{TRANSVERSE CONFINEMENT OF BEAM TAILS}

Considerations developed hereafter address the issue of environment irradiation relevant to high intensity hadron beams. It is shown how transverse beam tails can be manipulated, and possibly efficiently mastered, by means of the formalism described in Sec II.

It can be observed from geometrical representation of Eq. (2) [curves (1)-(5) in Fig. 5 as obtained with $n=$ $0-2$ as well as the top left-hand curve in Fig. 7] that there exists a critical value $y_{l c}^{\prime}$ of the starting angle at $s_{l}$, defined by the intersection of the asymptotic branch of the polynomial [Eq. (2)] with the $y= \pm \lambda_{1} y_{l}^{\prime}$ line, which can be expressed under the form

$$
(-)^{n} \lambda_{1}(s) y_{l c, 2 n+1}^{\prime}=\sum_{p=0}^{n} \lambda_{2 p+1}(s) y_{l c, 2 n+1}^{\prime 2 p+1} .
$$

That critical angle determines two regions in the incoming density $f\left(y_{l}^{\prime}\right)$ : Any particle with starting incidence $\left|y_{l}^{\prime}\right|<y_{l c, 2 n+1}^{\prime}$ (a contrario $\left|y_{l}^{\prime}\right|>y_{l c, 2 n+1}^{\prime}$ ) is confined at all $s>s_{l}$ within the region $|y(s)|<y_{c, 2 n+1}(s)$ (a contrario deconfined). In the case of sole octupole, Eqs. (2) and (12) lead to (Fig. 5)

$$
\begin{aligned}
y_{l c, 3}^{\prime} & =\sqrt{-2 \lambda_{1}(s) / \lambda_{3}(s)}, \\
y_{c, 3}(s) & =\left|\lambda_{1}(s)\right| \sqrt{-2 \lambda_{1}(s) / \lambda_{3}(s)},
\end{aligned}
$$

and in the octupole + dodecapole case they lead to

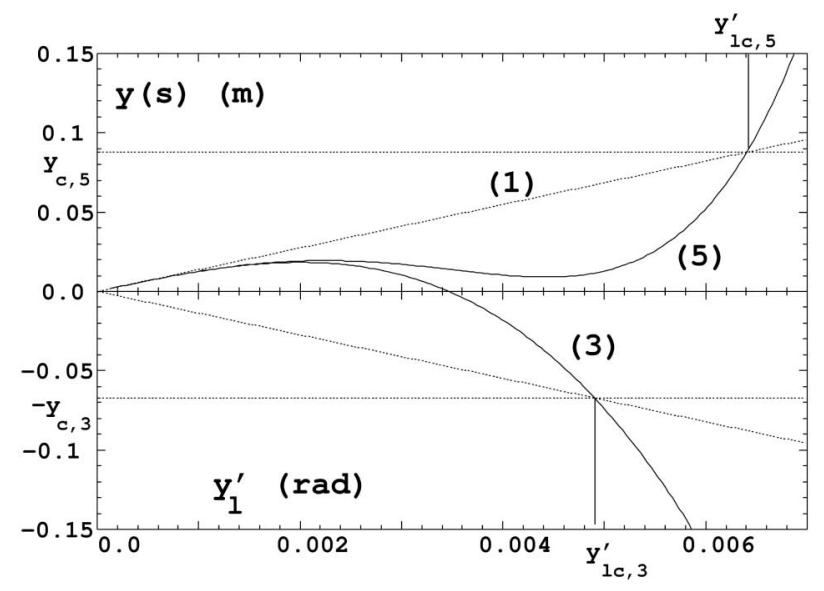

FIG. 5. Confinement critical angles $y_{l c, 2 n+1}^{\prime}(n=1,2)$. 


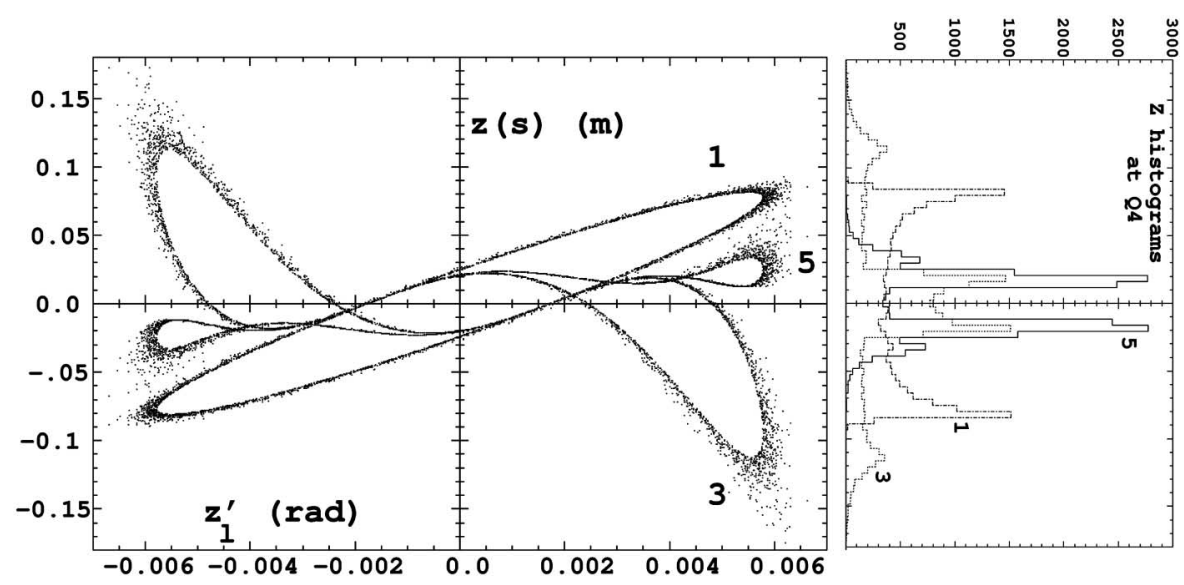

FIG. 6. Distortion of the elliptical phase space invariant and related histograms of the $\left[6 \sigma_{z}, 7 \sigma_{z}\right]$ region of the transverse beam tails at Q4.

$$
\begin{aligned}
y_{l c, 5}^{\prime} & =\sqrt{-\lambda_{3}(s) / \lambda_{5}(s)}, \\
y_{c, 5}(s) & =\left|\lambda_{1}(s)\right| \sqrt{\lambda_{3}(s) / \lambda_{5}(s)} .
\end{aligned}
$$

It ensues, in particular, that the superimposition of adequate high order components onto the octupole will decrease beam loss into chamber walls or, equivalently, will allow savings on transverse aperture of optical elements when designing the beam line (this, to some extent, may not be compatible with fifth order or higher order uniformization discussed earlier).

Figure 6 shows tracking results that illustrate the effect for the vertical motion in the Q4 quadrupole region [OV is the uniformization lens of concern, $y, y^{\prime}$ stand for $z, z^{\prime}$ in Eqs. (12)-(14)]: 4000 particles sorted at random in the range $\left[6 \sigma_{z^{\prime}}, 7 \sigma_{z^{\prime}}\right]$ of a Gaussian density $f\left(z_{l}^{\prime}\right)$ (relative population $<10^{-8}$ ) are launched from the upstream end of the uniformization lens $\mathrm{OV}$ and tracked down to Q4; the original vertical phase space ellipse at Q4 (curve 1, obtained with OV off) is distorted into curve 3 according to the polynomial transformation Eq. (2) in the octupole case, or into curve 5 in the octupole + dodecapole case; the $z$ extent of the distorted ellipse determines the nonlinear envelope $Z_{2 n+1}$ at Q4 as would be obtained from Eqs. (3)-(5). The related histograms in the right-hand figure show that a sole octupole at OV (curve 3 ) causes beam tail deconfinement with respect to the linear case (curve 1), whereas a combined octupole + dodecapole lens (curve 5) induces a strong confinement with respect to both former cases, as was already pointed out about beam envelopes (Figs. 1 and 3).

\section{CONCLUSION}

An analytical method for the calculation of nonlinear envelopes in beam expanders is presented; it has been implemented in a regular matrix transport code and provides fast and easy-to-handle means for beam line design and optimization. Some coupling effects (geometrical and momentum) may, to some extent, be accounted for. Consider- ations on transverse beam tail confinement are drawn and allow us to show that the use of high order nonlinearities with adequate strength permits decreasing particle loss and correlatively optical apertures along the expander.

\section{APPENDIX A: TRANSVERSE BEAM UNIFORMIZATION}

Transverse beam uniformization results from odd-order nonlinear kicks undergone by particles traveling off axis in the uniformization lens. The principle, in terms of beam density, can be understood on the basis of material derived in Ref. [5], as shown in Fig. 7: the transverse beam density $g[y(s)]$ (top right-hand curve) downstream of the uniformization lens builds up from the contributions of the $N \leq 2 n+1$ roots of Eq. (2) (whose geometrical representation is the top left-hand curve), whereas the integral $\int_{0}^{y} g(y) d y$ [Eq. (3)] builds up from the corresponding $N$ regions (shaded areas; $N=3$ in the present case) under the curve representative of the beam divergence $f\left(y_{l}^{\prime}\right)$ at the lens upstream end (bottom curve). The first folding in $y\left(y_{l}^{\prime}\right)$ at $y= \pm y_{M}$ (top left-hand curve) is caused by the octupole nonlinearity and causes confinement of the beam density $g[y(s)]$ within $\pm y_{M}$; the second folding at $y= \pm y_{m}$ is caused by the additional (yet not

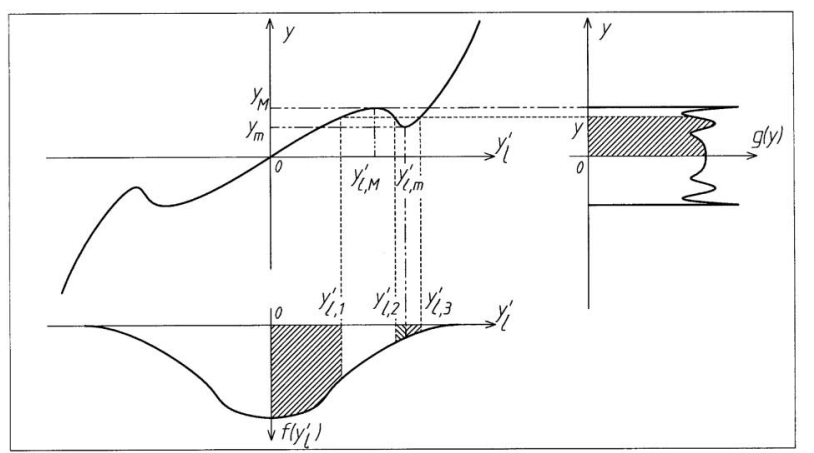

FIG. 7. A geometrical representation of the transverse density mapping upon polynomial transform Eq. (1), and of the integral Eq. (3). 
mandatory) dodecapole component whose main role as to uniformization is to increase particle population in the otherwise depressed regions $g\left( \pm y_{m}\right)$.

Beam uniformization can be achieved properly only if (we refer to the typical optical assembly introduced in Fig. 1)

(i) the transverse motions are uncoupled; in particular, the nonlinear lens $\mathrm{OH}$ (respectively, OV) devoted to the uniformization of the transverse horizontal (respectively, vertical) particle density distribution is to be placed at a waist of the vertical (respectively, horizontal) motion,

(ii) both the horizontal $\left(x, x^{\prime}\right)$ and vertical $\left(z, z^{\prime}\right)$ phasespace beam ellipses are flat at the upstream end of, respectively, the horizontal $(\mathrm{OH})$ and the vertical $(\mathrm{OV})$ uniformization nonlinear lens.

Given the first hypothesis, uniformization can be tuned independently in each plane. Given the second one, the beam ellipse whose general representation at arbitrary azimuth $s$ along the beam line is ( $y$ stands indifferently for $x$ or $z$ )

$$
\gamma(s) y^{2}+2 \alpha(s) y y^{\prime}+\beta(s) y^{\prime 2}=\epsilon_{y} / \pi
$$

[where $\alpha, \beta$, and $\gamma=\left(1+\alpha^{2}\right) / \beta$ are the local optical functions] will degenerate into

$$
y_{l}=r_{l} y_{l}^{\prime} \quad \text { with } r_{l}=-\beta_{l} / \alpha_{l}
$$

at the upstream of the nonlinear lens (a sufficient condition for a flat ellipse is $\sqrt{\beta_{l} \gamma_{l}} \approx \alpha_{l} \gg 1$ ). As a consequence, particle positions $y(s)$ at arbitrary $s>s_{l}$ downstream of a lens that combine $n$ nonlinear odd-order multipoles up to order $2 n+1$ are related to the angle $y_{l}^{\prime}$ at the upstream end of that lens by the polynomial relation Eq. (2), whose geometrical representation is the top left-hand curve in Fig. 7; in addition, beam phase space ellipses at all $s>s_{l}$ are filaments similar to the latter curve.

\section{APPENDIX B: TRANSVERSE BEAM DENSITY}

The transverse position $y(s)$ downstream of the lens and angle $y_{l}^{\prime}$ at the lens upstream end can be understood as random variables that describe the particle beam and therefore can be associated probability densities $g[y(s)]$ and $f\left(y_{l}^{\prime}\right)$ that satisfy the change of variable rule

$$
g(y)|d y|=\sum_{i=1}^{N} f\left(y_{l i}^{\prime}\right)\left|d y_{l i}^{\prime}\right|,
$$

wherein $y_{l i}^{\prime}(i=1, N)$ are the $N \leq 2 n+1$ real roots of Eq. (2), and, as such, functions of $y(s)$. Differentiation of Eq. (2) provides $d y$ that is further introduced in Eq. (B1), leading to Eq. (1).

\section{APPENDIX C: NONLINEAR TUNING OF THE BEAM LINE}

The transverse size $\pm y_{M}$ of the uniformized density $g[y(s)]$ (top right-hand plot in Fig. 7) is assured mostly by the octupole component in the nonlinear lens, with the constraint on the incoming beam divergence $\sigma_{y_{l}^{\prime}} \lesssim y_{l M}^{\prime}$ if $f$ is Gaussian $\left[y_{l M}^{\prime}\right.$ is the double root of Eq. (2) at $y=y_{M}$; that constraint is a third necessary condition for proper uniformization and a criterion for prior first order tuning of the expander $[4,5]]$.

From the hypothesis of a sole octupole component $(n=1)$ and considering that $\lambda_{1} \lambda_{3}<0$ for $\pm y_{M}$ real roots to Eq. (2) to exist, one gets the footprint size at target location $s=s_{T}, y_{M}=2 \lambda_{1}\left(s_{T}\right)\left[-\lambda_{1}\left(s_{T}\right) / 3 \lambda_{3}\left(s_{T}\right)\right]^{1 / 2} / 3$, from what the integrated octupole strength necessary for uniformized footprint at target ensues [noting $\Delta \phi_{T}=$ $\left.\phi\left(s_{T}\right)-\phi_{l}\right][5]$

$$
K_{3} L=\frac{4}{27 y_{M}^{2}} \frac{\beta\left(s_{T}\right)}{\beta_{l}^{2}} \frac{\cos ^{3} \Delta \phi_{T}}{\sin \Delta \phi_{T}} .
$$

An additional dodecapole mainly increases particle population around [10] $y_{m} \approx y_{M}\left(1+\sigma_{y_{l}^{\prime}}^{2} / y_{l M}^{\prime 2}\right) \times$ $\sqrt{1-2 \sigma_{y_{l}^{\prime}}^{2} / y_{l M}^{\prime 2}}$ (hence, a necessary condition $\sigma_{y_{l}^{\prime}}<$ $\left.y_{l M}^{\prime} / \sqrt{2}\right)$, which tends to smooth $g(y)$; this is equivalent to taking $\lambda_{1}\left(s_{T}\right) \lambda_{5}\left(s_{T}\right) \approx \lambda_{3}^{2}\left(s_{T}\right) / 4$, entailing an integrated strength in case of Gaussian incoming beam of [5]

$$
K_{5} L=-\frac{\left(K_{3} L\right)^{2}}{4} \beta_{l} \tan \Delta \phi_{T} .
$$

Note that effectiveness of the fifth order distortion requires that $f\left[y_{l}^{\prime}\left(y_{m}\right)\right]$ be non-negligible compared to $f\left[y_{l}^{\prime}\left(y<y_{M}\right)\right]$, which is hardly the case if $f$ is Gaussian and instead preaches for parabolic density as addressed in Ref. [1]. Eventually, uniformization can, in principle, be improved with additional higher order distortions, more or less successfully depending on the shape of the incoming density $f\left(y_{l}^{\prime}\right)$.

[1] See, for instance, B. Blind, Nucl. Instrum. Methods Phys. Res., Sect. B 56/57,1099-1102 (1991).

[2] D. P. Rusthoi et al., in Proceedings of the 17th International Linac Conference, Tsukuba, 1994 (KEK, Tsukuba, 1995).

[3] F. Méot, CEA-Saclay Report No. CEA/DSM/LNS/GECA/ GT/95-07, 1995.

[4] F. Méot and T. Aniel, CEA-Saclay Report No. CEA/ DSM/LNS/GECA/GT/95-05, 1995; in Proceedings of the 2nd BDO Workshop, St. Petersburg, Russia, 1995 (St. Petersburg University, St. Petersburg, Russia, 1996).

[5] F. Méot and T. Aniel, Nucl. Instrum. Methods Phys. Res., Sect. A 379, 196-205 (1996).

[6] F. Méot and T. Aniel, in Proceedings of EPAC'96, Sitges, Spain (Institute of Physics, Bristol, UK, 1996).

[7] F. Méot and T. Aniel, CEA-Saclay Report No. CEA/DSM/ DAPNIA/SEA-99-02, 1999.

[8] Transport code BETA, CEA/DSM/DAPNIA/SEA, CEASaclay, 91191 Gif-sur-Yvette Cedex, France.

[9] D. Bazin and B. M. Sherrill, Phys. Rev. E 50, 4017-4021 (1994).

[10] F. Méot and T. Aniel, CEA-Saclay Report No. CEA/DSM/ LNS/GECA/GT/95-05, 1995. 